\title{
Ruin Probabilities in a Risk Model with Two Types of Claims
}

\author{
Ji-Yeon $\mathrm{Han}^{1}$. Seung Kyoung Choi ${ }^{2}$. Eui Yong Lee ${ }^{3}$ \\ ${ }^{1}$ Department of Statistics, Sookmyung Women's University \\ ${ }^{2}$ Department of Statistics, Sookmyung Women's University \\ ${ }^{3}$ Department of Statistics, Sookmyung Women's University
}

(Received June 12, 2012; Revised July 11, 2012; Accepted August 28, 2012)

\begin{abstract}
A surplus process with two types of claims is considered, where Type I claims occur more frequently, however, their sizes are smaller stochastically than Type II claims. The ruin probabilities of the surplus caused by each type of claim are obtained by establishing integro-differential equations for the ruin probabilities. The formulas of the ruin probabilities contain an infinite sum and convolutions that make the formulas hard to be applicable in practice; subsequently, we obtain explicit formulas for the ruin probabilities when the sizes of both types of claims are exponentially distributed. Finally, we show through a numerical example, that Type II claims have more impact on the ruin probability of the surplus than Type I claims.
\end{abstract}

Keywords: Continuous time risk model, surplus process with two types of claims, ruin probability, integrodifferential equation.

\section{Introduction}

In this paper, we consider a variation of the classical continuous time risk model. The surplus in the model is initially at $u>0$, thereafter it increases linearly at a rate $c>0$ due to incoming premiums and decreases jumpwise due to arriving claims. There are two types of claims in the model. Type I claims arrive according to a Poisson process of rate $\lambda_{1}>0$ and sizes of claims are i.i.d. with distribution $G$, meanwhile, Type II claims arrive according to another Poisson process of rate $\lambda_{2}>0$ and sizes of claims are i.i.d. with distribution $H$. We assume that $\lambda_{1} \geq \lambda_{2}$ and distribution $H$ is stochastically larger than distribution $G$. That is, Type I claims occur more frequently, however, their sizes are smaller stochastically than Type II claims.

The surplus process of the model can be written as

$$
U(t)=u+c t-\sum_{i=1}^{N_{1}(t)} Y_{i}-\sum_{j=1}^{N_{2}(t)} Z_{j},
$$

This research was supported by Sookmyung Women's University Research Grants 2011.

${ }^{3}$ Corresponding author: Professor, Department of Statistics, Sookmyung Women's University, Seoul 140742, Korea. E-mail: eylee@sookmyung.ac.kr 


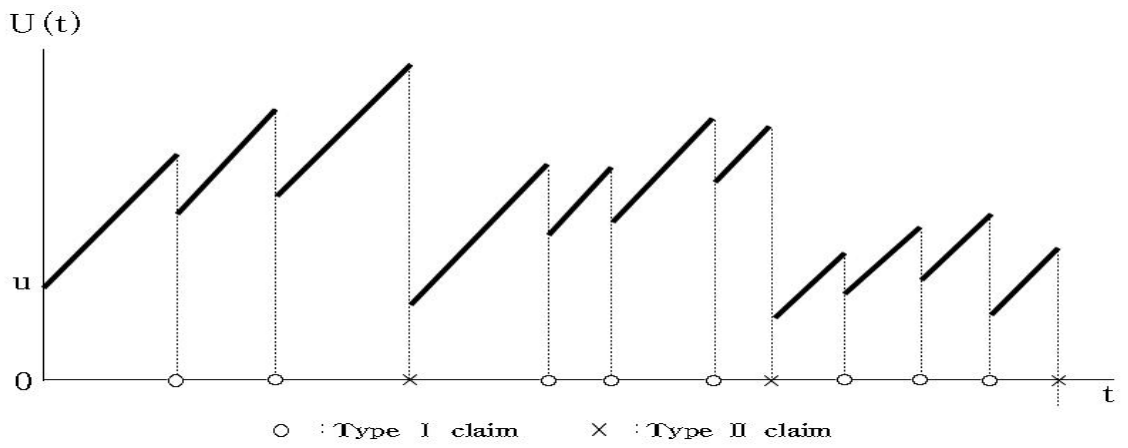

Figure 1.1. A sample path of $\{U(t), t \geq 0\}$

where $U(t)$ is the surplus at time $t>0, N_{1}(t)$ and $N_{2}(t)$ are the numbers of Type I and Type II claims up to time $t, Y_{i}$ and $Z_{j}$ denote the sizes of the $i^{\text {th }}$ Type I and the $j^{\text {th }}$ Type II claims with means $\mu_{1}$ and $\mu_{2}$, where $0<\mu_{1} \leq \mu_{2}$, and $c=(1+\theta)\left(\lambda_{1} \mu_{1}+\lambda_{2} \mu_{2}\right)$ is the premium rate, where $\theta>0$ is the relative security loading. A sample path of the surplus process in the model is illustrated in Figure 1.1. A ruin is said to occur if the surplus becomes zero or negative.

The ruin probabilities of the continuous time surplus process and its variations have been studied by many authors. The studies on the ruin probability of the surplus in the classical risk model is well summarized in Klugman et al. (2004). However, the ruin probability of the surplus even in the classical risk model contains an infinite sum and convolutions, hence, many approximations have been driven and well-known formulas were summarized and compared in Grandell (2000). Dufresne and Gerber (1991) considered a continuous time risk model where the surplus process was perturbed by diffusion and obtained the ruin probabilities. Tsai (2009) studied the ordering of the ruin probabilities in a similar model and obtained bounds on the ruin probabilities.

Recently, the risk model where there exist different types of claims was studied by several researchers. Chan et al. (2003) and Guo et al. (2007) studied the ruin probabilities in two-dimensional risk models. Chan et al. (2003) considered a bivariate risk model and derived a partial integro-differential equation satisfied by the two-dimensional ruin probabilities; however, the exact solution of the equation had not been obtained. Guo et al. (2007) considered two separate surplus processes and obtained a bound on the ruin probability of the sum of two surplus processes and bounds on the two-dimensional ruin probabilities of two separate surplus processes.

Li and Garrido (2005) and Lv et al. (2010) studied the ruin probabilities of the surplus in the risk model where there are two types of claims; claims of one type arrive according to a Poisson process and claims of the other type arrive according to a renewal process. Li and Garrido (2005) derived a Laplace transform of the non-ruin probability and obtained explicit results when the initial surplus is zero. Lv et al. (2010) obtained an exponential bound on the ruin probability. The formulas or the bounds of the ruin probability of the whole surplus process in the risk models have been studied by many authors; however, the exact ruin probabilities caused by each type of claim have not been derived yet. We, in this paper, obtain the formulas of the ruin probabilities caused by each type of claim by establishing integro-differential equations for the ruin probabilities.

In Section 2, we obtain the Laplace transforms of the ruin probabilities from the integro-differential equations and find the formulas of the ruin probabilities by inverting the Laplace transforms. These 
formulas contain an infinite sum and convolutions that makes the formulas hardly applicable to real insurance policies; subsequently, in Section 3, we obtain explicit formulas of the ruin probabilities, when the claim sizes of both types follow independently exponential distributions. These explicit formulas can be used as approximations to the true ruin probabilities in the case of general sizes of Type I and Type II claims. Finally, we give a numerical example to show that Type II claims contribute more to the ruin probability of the surplus than Type I claims, which is well recognized in the real world.

\section{Ruin Probabilities Caused by Each Type of Claim}

In this section, we, obtain the exact formulas of the ruin probabilities of the surplus caused by each type of claim, when the size distributions $(G$ and $H)$ of two types of claims are general. Let $\psi_{1}(u)$ be the probability of the ruin caused by a Type I claim, when the initial surplus is $u>0$. Observe that the following six mutually exclusive events can occur during a small interval $(0, h)$ :

(i) no claims occur, in which case

$$
\psi_{1}(u)=\psi_{1}(u+c h)
$$

(ii) a Type I claim occurs with $Y_{1} \geq u+c h^{\prime}\left(h^{\prime}<h\right)$, in which case

$$
\psi_{1}(u)=1
$$

(iii) a Type II claim occurs with $Z_{1} \geq u+c h^{\prime}\left(h^{\prime}<h\right)$, in which case

$$
\psi_{1}(u)=0
$$

(iv) a Type I claim occurs with $Y_{1}<u+c h^{\prime}$, in which case

$$
\psi_{1}(u)=\psi_{1}\left(u+c h^{\prime}-Y\right)
$$

(v) a Type II claim occurs with $Z_{1}<u+c h^{\prime}$, in which case

$$
\psi_{1}(u)=\psi_{1}\left(u+c h^{\prime}-Z\right)
$$

(vi) two or more claims occur; however, the probability of this event is $o(h)$.

From these relations, we can have

$$
\begin{aligned}
\psi_{1}(u)= & \left\{1-\left(\lambda_{1}+\lambda_{2}\right) h+o(h)\right\} \psi_{1}(u+c h) \\
& +\left\{\lambda_{1} h+o(h)\right\}\left\{1-\lambda_{2} h+o(h)\right\}\left\{1-G\left(u+c h^{\prime}\right)\right\} \times 1 \\
& +\left\{\lambda_{1} h+o(h)\right\}\left\{1-\lambda_{2} h+o(h)\right\} \int_{0}^{u+c h^{\prime}} \psi_{1}\left(u+c h^{\prime}-y\right) d G(y) \\
& +\left\{\lambda_{2} h+o(h)\right\}\left\{1-\lambda_{1} h+o(h)\right\} \int_{0}^{u+c h^{\prime}} \psi_{1}\left(u+c h^{\prime}-z\right) d H(z) \\
& +o(h) .
\end{aligned}
$$

After some algebra, dividing both sides by $c h$ and letting $h \rightarrow 0$ give the following integro-differential equation:

$$
-c \psi_{1}^{\prime}(u)=-\left(\lambda_{1}+\lambda_{2}\right) \psi_{1}(u)+\lambda_{1} \bar{G}(u)+\lambda_{1} \int_{0}^{u} \psi_{1}(u-y) d G(y)+\lambda_{2} \int_{0}^{u} \psi_{1}(u-z) d H(z),
$$

where $\bar{G}(u)=1-G(u)$. 
Let $\psi_{1}^{*}(s)=\int_{0}^{\infty} e^{-s u} \psi_{1}(u) d u$ be the Laplace transform of $\psi_{1}(u)$. Taking the Laplace transforms on both sides of Equation (2.1), we have

$$
\psi_{1}^{*}(s)=\frac{-c \psi_{1}(0)+\frac{\lambda_{1}}{s}\left\{1-g^{*}(s)\right\}}{-c s-\lambda_{1} g^{*}(s)-\lambda_{2} h^{*}(s)+\lambda_{1}+\lambda_{2}},
$$

where $g^{*}(s)=\int_{0}^{\infty} e^{-s u} d G(u)$ and $h^{*}(s)=\int_{0}^{\infty} e^{-s u} d H(u)$ are the Laplace-Stieltjes transform of $G$ and $H$, respectively. To find $\psi_{1}(0)$, we put $s \rightarrow 0$ in Equation $(2.2)$, then the denominator of $\psi_{1}^{*}(s)$ goes to zero. For $\psi_{1}^{*}(0)$ to exist, the numerator of $\psi_{1}^{*}(s)$ should also go to zero when $s \rightarrow 0$. This results in $\psi_{1}(0)=\lambda_{1} \mu_{1} / c$, since $\lim _{s \rightarrow 0}\left\{1-g^{*}(s)\right\} / s=-g^{*^{\prime}}(0)=\mu_{1}$.

To find $\psi_{1}(u)$, we, now, invert $\psi_{1}^{*}(s)$. Note that $\psi_{1}^{*}(s)$ can be written as

$$
\psi_{1}^{*}(s)=\frac{-\frac{\lambda_{1} \mu_{1}}{\lambda_{1} \mu_{1}+\lambda_{2} \mu_{2}}+\frac{\lambda_{1}}{\lambda_{1} \mu_{1}+\lambda_{2} \mu_{2}} \frac{1}{s}\left\{1-g^{*}(s)\right\}}{-\frac{\lambda_{1}}{\lambda_{1} \mu_{1}+\lambda_{2} \mu_{2}}+\frac{\lambda_{2}}{\lambda_{1} \mu_{1}+\lambda_{2} \mu_{2}}\left\{1-g^{*}(s)\right\}+\frac{\lambda_{1} \mu_{1}+\lambda_{2} \mu_{2}}{\lambda_{1}}\left\{1-h^{*}(s)\right\}} .
$$

Let $G_{e}(u)=1 / \mu_{1} \int_{0}^{u} \bar{G}(x) d x$ and $H_{e}(u)=1 / \mu_{2} \int_{0}^{u} \bar{H}(x) d x$ be the equilibrium distributions of $G$ and $H$, then it can be shown that the Laplace-Stieltjes transforms of $G_{e}$ and $H_{e}$ are given by

$$
\begin{aligned}
& g_{e}^{*}(s)=\int_{0}^{\infty} e^{-s u} d G_{e}(u)=\frac{1}{\mu_{1} s}\left\{1-g^{*}(s)\right\}, \\
& h_{e}^{*}(s)=\int_{0}^{\infty} e^{-s u} d H_{e}(u)=\frac{1}{\mu_{2} s}\left\{1-h^{*}(s)\right\} .
\end{aligned}
$$

Hence, $\psi_{1}^{*}(s)$ can be rewritten as

$$
\psi_{1}^{*}(s)=\frac{\frac{\lambda_{1} \mu_{1}}{c s}\left\{1-g_{e}^{*}(s)\right\}}{1-\left(\frac{\lambda_{1} \mu_{1}+\lambda_{2} \mu_{2}}{c}\right)\left\{\frac{\lambda_{1} \mu_{1}}{\lambda_{1} \mu_{1}+\lambda_{2} \mu_{2}} g_{e}^{*}(s)+\frac{\lambda_{2} \mu_{2}}{\lambda_{1} \mu_{1}+\lambda_{2} \mu_{2}} h_{e}^{*}(s)\right\}} .
$$

If we represent $\psi_{1}^{*}(s)$ as an infinite series, then

$$
\psi_{1}^{*}(s)=\frac{\lambda_{1} \mu_{1}}{c} \sum_{k=0}^{\infty}\left(\frac{1-g_{e}^{*}(s)}{s}\right)\left(\frac{\lambda_{1} \mu_{1}+\lambda_{2} \mu_{2}}{c}\right)^{k} \times\left(\frac{\lambda_{1} \mu_{1}}{\lambda_{1} \mu_{1}+\lambda_{2} \mu_{2}} g_{e}^{*}(s)+\frac{\lambda_{2} \mu_{2}}{\lambda_{1} \mu_{1}+\lambda_{2} \mu_{2}} h_{e}^{*}(s)\right)^{k} .
$$

Now, inverting Laplace transform, $\psi_{1}^{*}(s)$, gives

$$
\psi_{1}(u)=\frac{\lambda_{1} \mu_{1}}{c} \sum_{k=0}^{\infty}\left(\frac{\lambda_{1} \mu_{1}+\lambda_{2} \mu_{2}}{c}\right)^{k} \bar{G}_{e} \circ M^{(k)}(u),
$$

where o denotes the Stieltjes convolution, $(k)$ denotes the $k$-fold recursive Stieltjes convolution and

$$
M(u)=\frac{\lambda_{1} \mu_{1}}{\lambda_{1} \mu_{1}+\lambda_{2} \mu_{2}} G_{e}(u)+\frac{\lambda_{2} \mu_{2}}{\lambda_{1} \mu_{1}+\lambda_{2} \mu_{2}} H_{e}(u) .
$$

By symmetry, we can see that the probability of the ruin caused by a Type II claim is given by

$$
\psi_{2}(u)=\frac{\lambda_{2} \mu_{2}}{c} \sum_{k=0}^{\infty}\left(\frac{\lambda_{1} \mu_{1}+\lambda_{2} \mu_{2}}{c}\right)^{k} \bar{H}_{e} \circ M^{(k)}(u) .
$$




\section{Claims with Sizes Following Exponential Distributions}

In this section, we obtain explicit formulas of the ruin probabilities caused by each type of claim, when the claim sizes of both types follow independently exponential distributions, that is, when

$$
G(y)=1-e^{-\frac{y}{\mu_{1}}}, \quad \text { for } y>0 \quad \text { and } \quad H(z)=1-e^{-\frac{z}{\mu_{2}}}, \quad \text { for } z>0 .
$$

For the convenience of calculation, we, first, obtain $\phi_{1}(u)=1-\psi_{1}(u)$. From Equation (2.1), we can easily see that the integro-differential equation for $\phi_{1}(u)$ is given by

$$
\phi_{1}(u)=-\frac{\lambda_{2}}{c}+\left(\frac{\lambda_{1}+\lambda_{2}}{c}\right) \phi_{1}(u)+\frac{\lambda_{2}}{c} H(u)-\frac{\lambda_{1}}{c} \int_{0}^{u} \phi_{1}(u-y) d G(y)-\frac{\lambda_{2}}{c} \int_{0}^{u} \phi_{1}(u-z) d H(z) .
$$

Inserting $G(y)=1-e^{-y / \mu_{1}}$ and $H(z)=1-e^{-z / \mu_{2}}$ into the above equation and putting both $u-y$ and $u-z$ to be $x$ give

$$
\phi_{1}^{\prime}(u)=-\frac{\lambda_{2}}{\mu_{2} c} e^{-\frac{u}{\mu_{2}}}+\left(\frac{\lambda_{1}+\lambda_{2}}{c}\right) \phi_{1}(u)-\frac{\lambda_{1}}{\mu_{1} c} e^{-\frac{u}{\mu_{1}}} \int_{0}^{u} \phi_{1}(x) e^{\frac{x}{\mu_{1}}} d x-\frac{\lambda_{2}}{\mu_{2} c} e^{-\frac{u}{\mu_{2}}} \int_{0}^{u} \phi_{1}(x) e^{\frac{x}{\mu_{2}}} d x .
$$

To make Equation (3.1) solvable for $\phi_{1}(u)$, we eliminate both integral terms. To eliminate the last integral term, we differentiate Equation (3.1) with respect to $u$, then

$$
\begin{aligned}
\phi_{1}^{\prime \prime}(u)= & \frac{\lambda_{2}}{\mu_{2} c} e^{-\frac{u}{\mu_{2}}}+\left(\frac{\lambda_{1}+\lambda_{2}}{c}\right) \phi_{1}^{\prime}(u)-\frac{\lambda_{1}}{\mu_{1} c} \phi_{1}(u)-\frac{\lambda_{2}}{\mu_{2} c} \phi_{1}(u) \\
& +\frac{\lambda_{1}}{\mu_{1}^{2} c} e^{-\frac{u}{\mu_{1}}} \int_{0}^{u} \phi_{1}(x) e^{\frac{x}{\mu_{1}}} d x+\frac{\lambda_{2}}{\mu_{2}^{2} c} e^{-\frac{u}{\mu_{2}}} \int_{0}^{u} \phi_{1}(x) e^{\frac{x}{\mu_{2}}} d x .
\end{aligned}
$$

Multiplying Equation (3.1) by $1 / \mu_{2}$ and adding it with Equation (3.2) give

$$
\phi_{1}^{\prime \prime}(u)=\left(\frac{\lambda_{1}+\lambda_{2}}{c}-\frac{1}{\mu_{2}}\right) \phi_{1}^{\prime}(u)+\left(\frac{\lambda_{1}}{\mu_{2} c}-\frac{\lambda_{1}}{\mu_{1} c}\right) \phi_{1}(u)-\left(\frac{\lambda_{1}}{\mu_{1} \mu_{2} c}-\frac{\lambda_{1}}{\mu_{1}^{2} c}\right) e^{-\frac{u}{\mu_{1}}} \int_{0}^{u} \phi_{1}(x) e^{\frac{x}{\mu_{1}}} d x .
$$

Again, to eliminate the integral term in Equation (3.3), we differentiate Equation (3.3) with respect to $u$, then

$$
\begin{aligned}
\phi_{1}^{\prime \prime \prime}(u)= & \left(\frac{\lambda_{1}+\lambda_{2}}{c}-\frac{1}{\mu_{2}}\right) \phi_{1}^{\prime \prime}(u)+\left(\frac{\lambda_{1}}{\mu_{2} c}-\frac{\lambda_{1}}{\mu_{1} c}\right) \phi_{1}^{\prime}(u) \\
& -\left(\frac{\lambda_{1}}{\mu_{1} \mu_{2} c}-\frac{\lambda_{1}}{\mu_{1}^{2} c}\right) \phi_{1}(u)+\frac{1}{\mu_{1}}\left(\frac{\lambda_{1}}{\mu_{1} \mu_{2} c}-\frac{\lambda_{1}}{\mu_{1}^{2} c}\right) e^{-\frac{u}{\mu_{1}}} \int_{0}^{u} \phi_{1}(x) e^{\frac{x}{\mu_{1}}} d x .
\end{aligned}
$$

Multiplying Equation (3.3) by $1 / \mu_{1}$ and adding it with Equation (3.4), we, finally, have the following ordinary differential equation:

$$
\phi_{1}^{\prime \prime \prime}(u)=\left(\frac{\lambda_{1}+\lambda_{2}}{c}-\frac{1}{\mu_{1}}-\frac{1}{\mu_{2}}\right) \phi_{1}^{\prime \prime}(u)+\left(\frac{\lambda_{1}}{\mu_{2} c}+\frac{\lambda_{2}}{\mu_{1} c}-\frac{1}{\mu_{1} \mu_{2}}\right) \phi_{1}^{\prime}(u) .
$$

The general solution of Equation (3.5) is known to be

$$
\phi_{1}^{\prime}(u)=C_{1} e^{m_{1} u}+C_{2} e^{m_{2} u},
$$

where $m_{1}$ and $m_{2}$ are two solutions of

$$
m^{2}-\left(\frac{\lambda_{1}+\lambda_{2}}{c}-\frac{1}{\mu_{1}}-\frac{1}{\mu_{2}}\right) m-\left(\frac{\lambda_{1}}{\mu_{2} c}+\frac{\lambda_{2}}{\mu_{1} c}-\frac{1}{\mu_{1} \mu_{2}}\right)=0
$$


and they are

$$
\frac{\left(\frac{\lambda_{1}+\lambda_{2}}{c}-\frac{1}{\mu_{1}}-\frac{1}{\mu_{2}}\right) \pm \sqrt{\left(\frac{\lambda_{1}+\lambda_{2}}{c}-\frac{1}{\mu_{1}}-\frac{1}{\mu_{2}}\right)^{2}+4\left(\frac{\lambda_{1}}{\mu_{2} c}+\frac{\lambda_{2}}{\mu_{1} c}-\frac{1}{\mu_{1} \mu_{2}}\right)}}{2} .
$$

Observe that both solutions are real, since the inside of the squared root is equal to

$$
\left(\frac{\lambda_{1}-\lambda_{2}}{c}-\frac{1}{\mu_{1}}+\frac{1}{\mu_{2}}\right)^{2}+\frac{4 \lambda_{1} \lambda_{2}}{c^{2}}>0 .
$$

To evaluate $C_{1}$ and $C_{2}$, put $u=0$ in Equation (3.1) and Equation (3.3), while recalling that $\phi_{1}(0)=1-\psi_{1}(0)=1-\lambda_{1} \mu_{1} / c$, then

$$
\begin{aligned}
\phi_{1}^{\prime}(0) & =\left(\frac{\lambda_{1}+\lambda_{2}}{c}\right)\left(1-\frac{\lambda_{1} \mu_{1}}{c}\right)-\frac{\lambda_{2}}{c}, \\
\phi_{1}^{\prime \prime}(0) & =\left\{\left(\frac{\lambda_{1}+\lambda_{2}}{c}\right)^{2}-\frac{\lambda_{1}}{\mu_{1} c}\right\}\left(1-\frac{\lambda_{1} \mu_{1}}{c}\right)+\frac{\lambda_{1} \lambda_{2} \mu_{1}}{\mu_{2} c^{2}}-\frac{\lambda_{1} \lambda_{2}+\lambda_{2}^{2}}{c^{2}} .
\end{aligned}
$$

From these two boundary conditions, we can get $C_{1}$ and $C_{2}$, which are given by

$$
\begin{aligned}
C_{1}= & \frac{1}{m_{1}-m_{2}}\left\{\left(\frac{\lambda_{1}+\lambda_{2}}{c}\right)^{2}-\frac{\lambda_{1}}{\mu_{1} c}-\left(\frac{\lambda_{1}+\lambda_{2}}{c}\right) m_{2}\right\}\left(1-\frac{\lambda_{1} \mu_{1}}{c}\right) \\
& +\frac{1}{m_{1}-m_{2}}\left\{\frac{\lambda_{1} \lambda_{2}}{\mu_{2} c^{2}}\left(\mu_{1}-\mu_{2}\right)-\left(\frac{\lambda_{2}}{c}\right)^{2}+\frac{\lambda_{2} m_{2}}{c}\right\}, \\
C_{2}= & \frac{1}{m_{2}-m_{1}}\left\{\left(\frac{\lambda_{1}+\lambda_{2}}{c}\right)^{2}-\frac{\lambda_{1}}{\mu_{1} c}-\left(\frac{\lambda_{1}+\lambda_{2}}{c}\right) m_{1}\right\}\left(1-\frac{\lambda_{1} \mu_{1}}{c}\right) \\
& +\frac{1}{m_{2}-m_{1}}\left\{\frac{\lambda_{1} \lambda_{2}}{\mu_{2} c^{2}}\left(\mu_{1}-\mu_{2}\right)-\left(\frac{\lambda_{2}}{c}\right)^{2}+\frac{\lambda_{2} m_{1}}{c}\right\} .
\end{aligned}
$$

Integrating Equation (3.6), while recalling again that $\phi_{1}(0)=1-\lambda_{1} \mu_{1} / c$, gives

$$
\phi_{1}(u)=\frac{C_{1}}{m_{1}}\left(e^{m_{1} u}-1\right)+\frac{C_{2}}{m_{2}}\left(e^{m_{2} u}-1\right)+\left(1-\frac{\lambda_{1} \mu_{1}}{c}\right) .
$$

Finally, $\psi_{1}(u)=1-\phi_{1}(u)$.

By symmetry, the formula of $\psi_{2}(u)$ can be derived from that of $\psi_{1}(u)$ by interchanging $\lambda_{1}$ and $\lambda_{2}$, and also $\mu_{1}$ and $\mu_{2}$. We omit the details.

EXAMPLE 3.1. We numerically compare $\psi_{1}(u)$ and $\psi_{2}(u)$ to illustrate the influences of each type of claim to the ruin probability of the surplus in the risk model. It is assumed that $\lambda_{1} \mu_{1}=\lambda_{2} \mu_{2}$, that is, the expected total amount brought by Type I claims per unit time is same as that amount brought by Type II claims. Here, without loss of generality, we assume that $\lambda_{1} \mu_{1}=\lambda_{2} \mu_{2}=1$. It is also assumed that $u=10$ and $c=2.5$.

In Table 3.1, $\psi_{1}(u), \psi_{2}(u)$ and $\psi(u)$ are tabulated for various values of $\lambda_{2}$ and $\mu_{2}$, when both $\lambda_{1}$ and $\mu_{1}$ are given to be 1 . Here, $\psi(u)=\psi_{1}(u)+\psi_{2}(u)$ is the total ruin probability of the surplus 
Table 3.1. Ruin probabilities when $\lambda_{2}$ and $\mu_{2}$ are varying $\left(\lambda_{1}=\mu_{1}=1\right)$

\begin{tabular}{ccccc}
\hline$\lambda_{2}$ & $\mu_{2}$ & $\psi_{1}(u)$ & $\psi_{2}(u)$ & $\psi(u)$ \\
\hline 1 & 1 & 0.0541 & 0.0541 & 0.1083 \\
0.5 & 2 & 0.0632 & 0.1483 & 0.2115 \\
0.2 & 5 & 0.0512 & 0.3418 & 0.3930 \\
0.05 & 20 & 0.0200 & 0.5590 & 0.5790 \\
0.01 & 100 & 0.0053 & 0.6428 & 0.6481 \\
\hline
\end{tabular}

Table 3.2. Ruin probabilities when $\lambda_{1}$ and $\mu_{1}$ are varying $\left(\lambda_{2}=\mu_{2}=1\right)$

\begin{tabular}{ccccc}
\hline$\lambda_{1}$ & $\mu_{1}$ & $\psi_{1}(u)$ & $\psi_{2}(u)$ & $\psi(u)$ \\
\hline 1 & 1 & 0.0541 & 0.0541 & 0.1083 \\
1.5 & 0.67 & 0.0276 & 0.0458 & 0.0734 \\
2 & 0.50 & 0.0172 & 0.0405 & 0.0577 \\
5 & 0.20 & 0.0044 & 0.0302 & 0.0347 \\
10 & 0.10 & 0.0018 & 0.0270 & 0.0287 \\
\hline
\end{tabular}

Table 3.3. Ruin probabilities when $\lambda_{2}$ and $\mu_{2}$ are varying $\left(\lambda_{1}=\mu_{1}=1\right)$

\begin{tabular}{ccccc}
\hline$\lambda_{2}$ & $\mu_{2}(=\alpha)$ & $\psi_{1}(u)$ & $\psi_{2}(u)$ & $\psi(u)$ \\
\hline 1 & 1 & 0.0615 & 0.0620 & 0.1235 \\
0.5 & 2 & 0.0740 & 0.0935 & 0.1675 \\
0.2 & 5 & 0.0560 & 0.2465 & 0.3025 \\
0.05 & 20 & 0.0255 & 0.5275 & 0.5530 \\
0.01 & 100 & 0.0035 & 0.6535 & 0.6570 \\
\hline
\end{tabular}

caused by either type of claim.

We can see that $\psi_{2}(u) \geq \psi_{1}(u)$, that is, Type II claims have more impact on the ruin probability of the surplus than Type I claims. We can also see that $\psi_{2}(u)$ increases rapidly as $\mu_{2}$ increases even though $\lambda_{2}$ decreases, which results in, consequently, the rapid increase of $\psi(u)$.

In Table 3.2, $\psi_{1}(u), \psi_{2}(u)$ and $\psi(u)$ are tabulated for various values of $\lambda_{1}$ and $\mu_{1}$, when both $\lambda_{2}$ and $\mu_{2}$ are given to be 1 .

Again, we can see that $\psi_{2}(u) \geq \psi_{1}(u)$. It can be also seen that $\psi_{1}(u)$ as well as $\psi_{2}(u)$ decrease as $\mu_{1}$ decreases even though $\lambda_{1}$ increases; however, $\psi_{2}(u)$ decreases relatively slowly compared to $\psi_{1}(u)$. These facts repeatedly show that the sizes of claims have more impact on the ruin probability of the surplus than the frequencies of claims.

EXAMPLE 3.2. Since the formulas of $\psi_{1}(u)$ and $\psi_{2}(u)$ in Equation (2.3) and Equation (2.4) contain infinite sums and Stieltjes convolutions, it is hard to evaluate $\psi_{1}(u)$ and $\psi_{2}(u)$ when $G$ and $H$ are general distributions other than exponential distributions. However, we evaluate $\psi_{1}(u)$ and $\psi_{2}(u)$ by simulation when $G$ and $H$ are gamma distributions with shape parameter $\alpha$ and scale parameter $\beta=1$, to see again the influences of each type of claim to the ruin probability of the surplus. It is also assumed that $\lambda_{1} \mu_{1}=\lambda_{2} \mu_{2}=1$ to make the expected total amount brought by Type I claims per unit time be equal to that brought by Type II claims.

$\psi_{1}(u), \psi_{2}(u)$ and $\psi(u)$ are tabulated in Table 3.3 for various values of $\lambda_{2}$ and $\mu_{2}$ when $\lambda_{1}=\mu_{1}=1$ and $\psi_{1}(u), \psi_{2}(u)$ and $\psi(u)$ are tabulated in Table 3.4 for various values of $\lambda_{1}$ and $\mu_{1}$ when $\lambda_{2}=$ $\mu_{2}=1$. Table 3.3 and Table 3.4 show very similar patterns to those in Table 3.1 and Table 3.2. That 
Table 3.4. Ruin probabilities when $\lambda_{1}$ and $\mu_{1}$ are varying $\left(\lambda_{2}=\mu_{2}=1\right)$

\begin{tabular}{ccccc}
\hline$\lambda_{1}$ & $\mu_{1}(=\alpha)$ & $\psi_{1}(u)$ & $\psi_{2}(u)$ & $\psi(u)$ \\
\hline 1 & 1 & 0.0615 & 0.0620 & 0.1235 \\
1.5 & 0.67 & 0.0355 & 0.0590 & 0.0945 \\
2 & 0.50 & 0.0325 & 0.0500 & 0.0825 \\
5 & 0.20 & 0.0240 & 0.0485 & 0.0725 \\
10 & 0.10 & 0.0150 & 0.0460 & 0.0610 \\
\hline
\end{tabular}

is, Type II claims with lower frequency but larger size have more impact on the ruin probability of the surplus than Type I claims with higher frequency but smaller size.

\section{References}

Chan, W. S., Yang, H. and Zhang, L. (2003). Some results on ruin probabilities in a two-dimensional risk model, Insurance: Mathematics \& Economics, 32, 345-358.

Dufresne, F. and Gerber, H. U. (1991). Risk theory for the compound Poisson process that is perturbed by diffusion, Insurance: Mathematics \& Economics, 10, 51-59.

Grandell, J. (2000). Simple approximations of the ruin probabilities, Insurance: Mathematics and Economics, 26, 157-173.

Guo, J. Y., Yuen, K. C. and Zhou, M. (2007). Ruin probabilities in Cox risk models with two dependent classes of business, Acta Mathematica Sinica, English Series, 23, 1281-1288.

Klugman, S. A., Panjer, H. H. and Willmot, G. E. (2004). Loss Model: From Data to Decisions, 2nd ed, John Wiley \& Sons, Hoboken.

Li, S. and Garrido, J. (2005). Ruin probabilities for two classes of risk processes, ASTIN Bulletin, 35, 61-77.

Lv, T. L., Guo, J. Y. and Zhang, X. (2010). Ruin probabilities for a risk model with two classes of claims, Acta Mathematica Sinica, English Series, 26, 1749-1760.

Tsai, C. C. L. (2009). On the ordering of ruin probabilities for the surplus process perturbed by diffusion, Scandinavian Actuarial Journal, 2009, 187-204. 